\title{
Proton Beam Therapy for Hepatocellular Carcinoma after the Fontan Procedure
}

\author{
Haruko Numajiri1 ${ }^{*}$, Masashi Mizumoto¹, Toshiyuki Okumura1, Nobuyoshi Fukumitsu², \\ Naoyuki Hasegawa3 ${ }^{3}$ Kazunori Ishige ${ }^{4}$, Kuniaki Fukuda ${ }^{3,4}$, Tomoko Ishizu ${ }^{5}$, \\ Katsutoshi Tokushige ${ }^{6}$, Hideyuki Sakurai ${ }^{1}$
}

\footnotetext{
${ }^{1}$ Department of Radiation Oncology, Proton Medical Research Center, University of Tsukuba Hospital, Tsukuba, Japan

${ }^{2}$ Department of Radiation Oncology, Kobe Proton Center, Kobe, Japan

${ }^{3}$ Department of Gastroenterology, University of Tsukuba Hospital, Tsukuba, Japan

${ }^{4}$ Department of Gastroenterology, Kasumigaura Medical Center, Tsuchiura, Japan

${ }^{5}$ Department of Cardiology, University of Tsukuba Hospital, Tsukuba, Japan

${ }^{6}$ Department of Internal Medicine, Institute of Gastroenterology, Tokyo Women's Medical University, Tokyo, Japan

Email: ^haruko@pmrc.tsukuba.ac.jp
}

How to cite this paper: Numajiri, $\mathrm{H}$. Mizumoto, M., Okumura, T., Fukumitsu, N., Hasegawa, N., Ishige, K., Fukuda, K., Ishizu, T., Tokushige, K. and Sakurai, H. (2021) Proton Beam Therapy for Hepatocellular Carcinoma after the Fontan Procedure. Journal of Cancer Therapy, 12, 554-562.

https://doi.org/10.4236/jct.2021.1210046

Received: September 13, 2021

Accepted: October 18, 2021

Published: October 21, 2021

Copyright $\odot 2021$ by author(s) and Scientific Research Publishing Inc. This work is licensed under the Creative Commons Attribution International License (CC BY 4.0).

http://creativecommons.org/licenses/by/4.0/ (c) (i) Open Access

\begin{abstract}
Background: Hepatocellular carcinoma (HCC) is more likely to occur in patients with a history of Fontan surgery, possibly due to long-term liver congestion. Proton beam therapy (PBT) may be effective for HCC that develops after Fontan surgery. Methods: Six lesions in 5 patients ( 3 females, 2 males) received PBT. The median age of the patients was 33 (range $21-42$ ) years, and the median age at the time of the Fontan procedure was $6(5-13)$ years. Four patients had multiple HCC at the time of PBT. The median tumor size was $57(22-80) \mathrm{mm}$ and 4 patients were classified as Child-Pugh B. Two patients received transcatheter arterial chemoembolization before PBT. The schedule of PBT was $66 \mathrm{~Gy}$ (RBE) in 10 fractions for 2 lesions, $72.6 \mathrm{~Gy}$ (RBE) in 22 fractions for 2 lesions, and $74 \mathrm{GyE}$ (RBE) in 37 fractions for 2 lesions. Results: The median follow-up period was 31 (10 - 46) months, and the numbers of survivors, deaths from primary diseases, and deaths from other diseases were 3,1 , and 1 , respectively. There were no local recurrences, one intrahepatic metastasis, one lung metastasis, and one intrathoracic metastasis. Conclusion: Although experiences on a small number of patients cannot conclude things, we believe that PBT can be a reasonable choice of radical treatment for HCC occurring after the Fontan procedure.
\end{abstract}

\section{Keywords}

Radiotherapy, Proton Beam Therapy, Hepatocellular Carcinoma, Fontan Procedure 


\section{Introduction}

A Fontan procedure is a palliative operation for patients with single ventricle congenital heart disease (CHD). Since it was first described in 1971, the Fontan procedure has been widely accepted for use in children [1] [2]. However, many reports have shown that Fontan-associated liver disease (FALD) occurs due to hepatic venous congestion and ischemia [2] [3] [4] [5], and some recent reports have suggested that the risk of hepatocellular carcinoma (HCC) is higher after a Fontan procedure [6] [7] [8] [9] [10].

The standard curative treatments for HCC are surgery, liver transplantation, radiofrequency ablation (RFA) and transarterial chemoembolization (TACE). However, these are often difficult to perform for HCC after a Fontan procedure because of low heart function, hypohepatic function, and liver congestion. We have shown that proton beam therapy (PBT) can be performed for patients with a poor general condition [11] [12] [13] [14]. Herein, we describe the first use of PBT for HCC after a Fontan procedure.

\section{Methods}

From 2016 to 2018, we performed PBT for 5 patients ( 3 females, 2 males) with HCC after a Fontan procedure. This retrospective study was approved by the institutional review board and written informed consent was obtained from all patients. The median age of the patients was 33 (range 21 - 42) years, and the median age at the time of the Fontan procedure was 6 (range 5 -13) years. The Fontan procedure was performed for double outlet right ventricle $(\mathrm{n}=3)$ and tricuspid atresia $(n=2)$. Four patients needed anticoagulation therapy after the Fontan procedure.

At the time of PBT, 4 patients had multiple HCC. Two patients had received TACE before PBT. The median time from the Fontan procedure to PBT was 26 (15 - 33) years. Six lesions in 5 patients received PBT because standard treatment was considered to be difficult. The median tumor size was $57(22-80) \mathrm{mm}$ and 4 patients were classified as Child-Pugh B. Three treatment schedules were used: $66 \mathrm{~Gy}$ (RBE) in 10 fractions for 2 lesions, $72.6 \mathrm{~Gy}$ (RBE) in 22 fractions for 2 lesions, and $74 \mathrm{GyE}$ (RBE) in 37 fractions for 2 lesions. These were selected based on the tumor location.

\section{Results}

The backgrounds of the patients and the tumor characteristics are shown in Table 1. Each PBT schedule was performed without interruption. One patient had grade 4 hyperkalemia, which was probably a side effect of a diuretic. No other grade 3 or higher acute toxicity was observed. At the time of analysis, three patients are alive, one died due to heart failure, and one died due to bleeding from intrathoracic metastasis. The median follow-up period was 31 months (range: 10 - 46 months). There has been no local recurrence, but one case has had intrahepatic recurrence outside the irradiated field, one developed lung metastasis, and one had intrathoracic metastasis. 
Table 1. Patients and tumor characteristics $(n=5)$.

\begin{tabular}{|c|c|}
\hline Item & Value \\
\hline Age at PBT, years (median) & $21-42(33)$ \\
\hline Age at Fontan procedure & $5-13(6)$ \\
\hline Sex (male/female) & $2 / 3$ \\
\hline \multicolumn{2}{|l|}{ Heart disease } \\
\hline Double outlet right ventricle & 3 \\
\hline Tricuspid atresia & 2 \\
\hline \multicolumn{2}{|l|}{$\mathrm{HCC}$ at $\mathrm{PBT}$} \\
\hline Single & 1 \\
\hline Multiple & 4 \\
\hline Time from Fontan procedure to PBT (years) & $15-33(26)$ \\
\hline \multicolumn{2}{|l|}{ Child-Pugh classification } \\
\hline A & 1 \\
\hline B & 4 \\
\hline \multicolumn{2}{|l|}{ Proton therapy dose, $G y(R B E): 6$ lesions } \\
\hline $66 \mathrm{GyE} / 10 \mathrm{Fr}$ & 2 \\
\hline $72.6 \mathrm{GyE} / 22 \mathrm{Fr}$ & 2 \\
\hline $74 \mathrm{GyE} / 37 \mathrm{Fr}$ & 2 \\
\hline \multicolumn{2}{|l|}{ Anticoagulant or antiplatelet } \\
\hline Yes & 4 \\
\hline No & 1 \\
\hline
\end{tabular}

PBT: Proton beam therapy; HCC: Hepatocellular carcinoma; RBE: Relative biological effectiveness.

As an illustrative example, we describe the case of a 33-year-old Asian female who was born with tricuspid atresia. At the age of 6, she underwent a Fontan procedure followed by a total cavopulmonary connection at age 23 . She had been under observation by abdominal US and CT from her twenties, and a dynamic CT image revealed multiple HCC in segment 7 at age 32 (Figure 1(b), Figure 1(c)). The HCCs were $5.5 \mathrm{~cm}$ and $2.9 \mathrm{~cm}$ in diameter and both protruded on the liver surface, but there was no vascular invasion. Her serum alpha-fetoprotein level increased to $9883 \mathrm{ng} / \mathrm{ml}$ and PIVKAII also increased to $33,290 \mathrm{mAU} / \mathrm{ml}$ with anticoagulant. Liver function was not good, at Child-Pugh B class, with medium retention on an ICG test (retention rate at $15 \mathrm{~min}, 23 \%$ ). Heart function was also not good, with atrial flutter, tachycardia-bradycardia syndrome, and coronary sinus thrombosis. The patient refused a surgical approach because of the risks of liver and heart failure. And RFA or TACE were also thought to be risky because of use of anticoagulant. For these reasons, she was referred to our PBT center. 


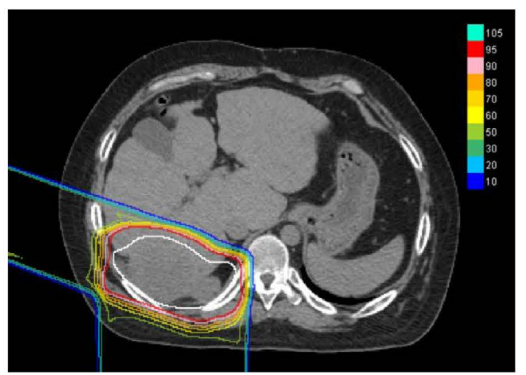

(a)

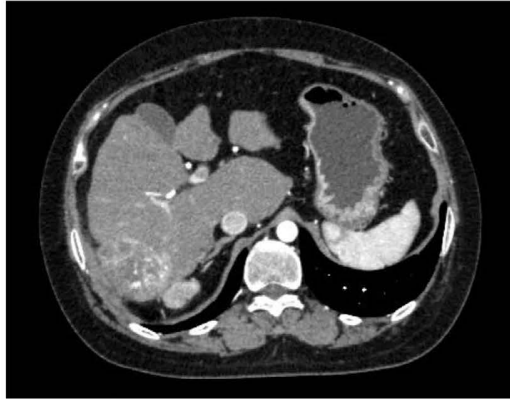

(b)

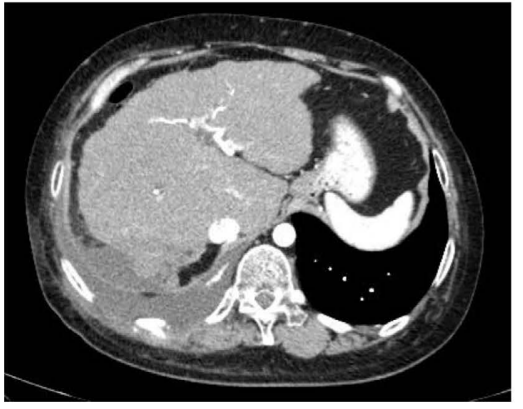

(d)

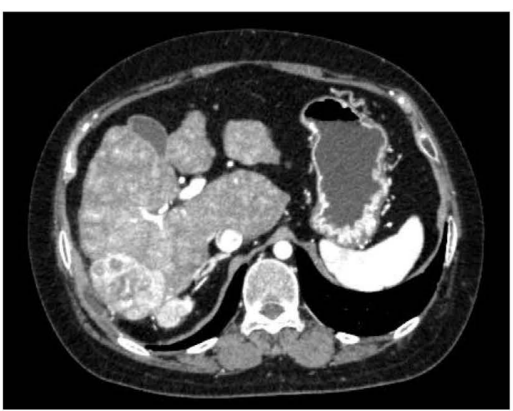

(c)

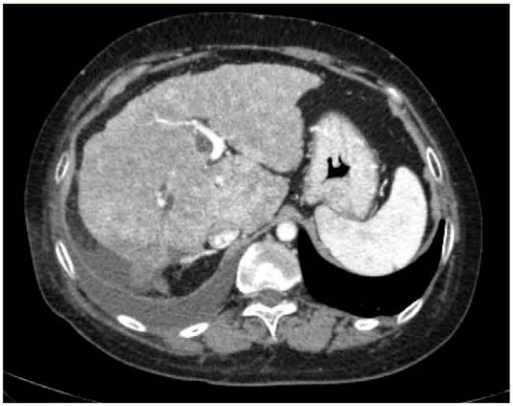

(e)

Figure 1. (a) Treatment plan for proton beam therapy with an isodose distribution in an axial view, showing isodose curves representing $100 \%$ to $10 \%$ of the prescribed dose at $10 \%$ intervals; (b), (c) CT showing HCC in the right posterior segment of the liver before proton beam therapy. (b) Arterial phase. (c) Venous phase; (d), (e) CT showing that HCC had disappeared 31 months after proton beam therapy. (d) Arterial phase. (e) Venous phase.

A total dose of $72.6 \mathrm{GyE}$ in 22 fractions for 5 weeks was delivered to the tumor (Figure 1(a)) without any significant adverse events. At 5 months after PBT, CT revealed multiple lung metastases. Sorafenib, gefitinib, and paclitaxel were administered while the effects of chemotherapy were monitored, and at 31 months after PBT the patient is alive with the disease. The HCCs in her liver are shrunk and stable (Figure 1(d), Figure $1(\mathrm{e})$ ) and there have been no adverse events of $>\mathrm{G} 3$.

\section{Discussion}

In 1971, Fontan reported the basic surgery in the Fontan procedure [1]. Subse- 
quently, the surgical technique has been improved, and since the extracardiac conduit method using an artificial blood vessel was reported in 1990, stable postoperative management has become possible and the number of treated cases has increased [2]. However, the number of reports of postoperative complications has also increased since the 1990s [3] [4] and various measures for complications and follow-up methods have been proposed [5] [15] [16]. One complication after Fontan surgery is an increased incidence of HCC, which several reports since 2010 have described [7] [8] [17] [18] [19] (Table 2). In these reports, the incidence of HCC after Fontan surgery is $0.18 \%$ to $1.4 \%$, which is higher than the prevalence of HCC in the general population of Japan. Furthermore, the rate of HCC is $2.3 \%$ to $4.4 \%$ in the limited number of cases in which imaging results are available after Fontan surgery.

From 2010 to 2018, 34 cases of HCC after Fontan surgery have been described in 18 reports [8] [19] [20]-[36] (Table 3). The median age at onset of HCC of 28

Table 2. Hepatocellular carcinoma incidence after Fontan procedure.

\begin{tabular}{cccc}
\hline Author, year & $\mathrm{n}$ & Group & Incident rate \\
\hline Elder, 2013 & 73 & All patients & 1.4 \\
Wallihan, 2013 & 42 & Followed by imaging & 2.3 \\
Pundi, 2016 & 1138 & All patients & 0.44 \\
Kuwabara, 2018 & 195 & Followed by imaging & 2.6 \\
Nandwana, 2018 & 2700 & All patients & 0.18 \\
\hline
\end{tabular}

Table 3. Summary of 34 cases (18 reports) on HCC after a Fontan procedure.

\begin{tabular}{cc}
\hline Item & Value \\
\hline $\begin{array}{c}\text { Age at treatment for HCC (years) } \\
\text { From Fontan procedure to HCC (years) }\end{array}$ & $12-51(29)$ \\
Hepatitis & $7-35(22)$ \\
Hepatitis C & 3 \\
None & 31 \\
Treatment method & \\
Surgery & 7 \\
TAE/TACE & 9 \\
RFA & 6 \\
Chemotherapy & 5 \\
Best supportive care & 6 \\
No information & 1 \\
\hline
\end{tabular}

TAE: Transcatheter arterial embolization; TACE: Transcatheter arterial chemo-embolization; RFA: Radiofrequency ablation. 
years indicates that this disease mainly affects young people. After Fontan surgery, patients often need to take anticoagulants or have a pacemaker in place to maintain cardiac function or normal blood flow, and thus, the treatment methods for HCC are limited. Of the 34 reported cases, 11 had difficulty with curative treatment.

PBT has been established as a radical treatment for HCC, and can be used as a radical therapeutic method even in cases with low liver function or those in which the inferior vena cava or portal vein has a tumor plug. All 5 cases in which we used PBT were judged to be difficult to cure radically, but in all cases, radical PBT was completed according to the protocol and without any serious adverse events to date. In addition, PBT reduces the risk of secondary cancer in pediatric cases, and this effect can be expected in patients with a young onset age of HCC after a Fontan procedure.

HCC is a heterogeneous population that can be triggered by several clinical backgrounds.

Therefore, depending on the etiology of each, the HCCs that develop may have different cytological and oncological characteristics. It is not yet clear what the cytological characteristics of HCC after Fontan surgery are, and whether proton therapy is advantageous for these characteristics. However, it may be a relatively reasonable treatment to receive, at least for those with poor liver and circulatory system conditions.

\section{Conclusion}

This is the first report of the use of PBT for HCC after a Fontan procedure. The results show that $\mathrm{PBT}$ has potential as a radical treatment in the special circumstances present in this population of patients.

\section{Conflicts of Interest}

The authors declare that they have no competing interests.

\section{Financial Disclosure}

All authors have no financial relationships associated with the content of the manuscript.

\section{Availability of Data and Materials}

Please contact the corresponding author for data requests.

\section{Ethics Approval and Consent to Participate}

The patients signed a consent form prior to initiation of treatment and collection of data.

\section{Authorization Number of This Study}

H28-152; authorized by Ethics Committee University of Tsukuba Hospital. 


\section{References}

[1] Fontan, F. and Baudet, E. (1971) Surgical Repair of Tricuspid Atresia. Thorax, 26, 240-248. https://doi.org/10.1136/thx.26.3.240

[2] Davies, R.R., Chen, J.M. and Mosca, R.S. (2011) The Fontan Procedure: Evolution in Technique; Attendant Imperfections and Transplantation for "Failure". Seminars in Thoracic and Cardiovascular Surgery: Pediatric Cardiac Surgery Annual, 14, 55-66. https://doi.org/10.1053/j.pcsu.2011.01.014

[3] Wu, F.M., Ukomadu, C., Odze, R.D., Valente, A.M., Mayer, J.E. and Earing, M.G. (2011) Liver Disease in the Patient with Fontan Circulation. Congenital Heart Disease, 6, 190-201. https://doi.org/10.1111/j.1747-0803.2011.00504.x

[4] Ohuchi, H. (2016) Adult Patients with Fontan Circulation: What We Know and How to Manage Adults with Fontan Circulation? Journal of Cardiology, 68, 181-189. https://doi.org/10.1016/j.jjcc.2016.04.001

[5] Hilscher, M.B., Johnson, J.N., Cetta, F., Driscoll, D.J., Poterucha, J.J., Sanchez, W., Connolly, H.M. and Kamath, P.S. (2017) Surveillance for Liver Complications after the Fontan Procedure. Congenital Heart Disease, 12, 124-132. https://doi.org/10.1111/chd.12446

[6] Ho, S.S., Brown, R. and Fitzgibbon, B. (1990) Hepatocellular Carcinoma with Cardiac Cirrhosis. The Medical Journal of Australia, 152, 553-554.

https://doi.org/10.5694/j.1326-5377.1990.tb125362.x

[7] Pundi, K., Pundi, K.N., Kamath, P.S., Cetta, F., Li, Z., Poterucha, J.T., Driscoll, D.J. and Johnson, J.N. (2016) Liver Disease in Patients after the Fontan Operation. American Journal of Cardiology, 117, 456-460.

https://doi.org/10.1016/j.amjcard.2015.11.014

[8] Kuwabara, M., Niwa, K., Toyoda, T., Shirai, T., Tateno, S., Ohuchi, H., Tanaka, Y., Ichida, F., Fujisawa, T., Akagi, T., Mori, Y. and Research Committee of the Japanese Society of Pediatric Cardiology and Cardiac Surgery (2018) Liver Cirrhosis and/or Hepatocellular Carcinoma Occurring Late after the Fontan Procedure-A Nationwide Survey in Japan. Circulation Journal, 82, 1155-1160.

https://doi.org/10.1253/circj.CJ-17-1053

[9] Possner, M., Gordon-Walker, T., Egbe, A.C., Poterucha, J.T., Warnes, C.A., Connolly, H.M., Ginde, S., Clift, P., Kogon, B., Book, W.M., Walker, N., Wagenaar, L.J., Moe, T., Oechslin, E., Kay, W.A., Norris, M., Dillman, J.R., Trout, A.T., Anwar, N., Hoskoppal, A., Broering, D.C., Bzeizi, K. and Veldtman, G. (2020) Hepatocellular Carcinoma and the Fontan Circulation: Clinical Presentation and Outcomes. International Journal of Cardiology, 322, 142-148.

[10] Sagawa, T., Kogiso, T., Sugiyama, H., Hashimoto, E., Yamamoto, M. and Tokushige, K. (2020) Characteristics of Hepatocellular Carcinoma Arising from Fontan-Associated Liver Disease. Hepatology Research, 50, 853-862. https://doi.org/10.1111/hepr.13500

[11] Hata, M., Tokuuye, K., Sugahara, S., Kagei, K., Igaki, H., Hashimoto, T., Ohara, K., Matsuzaki, Y., Tanaka, N. and Akine, Y. (2005) Proton Beam Therapy for Hepatocellular Carcinoma with Portal Vein Tumor Thrombus. Cancer, 104, 794-801. https://doi.org/10.1002/cncr.21237

[12] Hata, M., Tokuuye, K., Sugahara, S., Tohno, E., Nakayama, H., Fukumitsu, N., Mizumoto, M., Abei, M., Shoda, J., Minami, M. and Akine, Y. (2007) Proton Beam Therapy for Aged Patients with Hepatocellular Carcinoma. International Journal of Radiation Oncology, Biology, Physics, 69, 805-812.

https://doi.org/10.1016/j.ijrobp.2007.04.016 
[13] Hata, M., Tokuuye, K., Sugahara, S., Fukumitsu, N., Hashimoto, T., Ohnishi, K., Nemoto, K., Ohara, K., Matsuzaki, Y. and Akine, Y. (2006) Proton Beam Therapy for Hepatocellular Carcinoma with Limited Treatment Options. Cancer, 107, 591-598. https://doi.org/10.1002/cncr.22039

[14] Mizumoto, M., Oshiro, Y., Okumura, T., Fukumitsu, N., Numajiri, H., Ohnishi, K., Aihara, T., Ishikawa, H., Tsuboi, K. and Sakurai, H. (2016) Proton Beam Therapy for Hepatocellular Carcinoma: A Review of the University of Tsukuba Experience. International Journal of Particle Therapy, 2, 570-578. https://doi.org/10.14338/IJPT-15-00035.2

[15] Wells, M.L., Hough, D.M., Fidler, J.L., Kamath, P.S., Poterucha, J.T. and Venkatesh, S.K. (2017) Benign Nodules in Post-Fontan Livers Can Show Imaging Features Considered Diagnostic for Hepatocellular Carcinoma. Abdominal Radiology, 42, 2623-2631. https://doi.org/10.1007/s00261-017-1181-9

[16] Wilson, T.G., d’Udekem, Y., Winlaw, D.S., Cordina, R.L., Celermajer, D.S., Wheaton, G.R., Bullock, A., Gentles, T.L., Weintraub, R.G., Justo, R.N., Grigg, L.E., Radford, D.J., Hardikar, W., Cheung, M., Cain, T.M., Rao, P., Alexander, S.I., Ayer, J., Verrall, C., Du Plessis, K., Chapman, J., Rice, K., Barry, J., Zannino, D. and Iyengar, A.J. (2018) Australian and New Zealand Fontan Registry. Hepatic and Renal End-Organ Damage in the Fontan Circulation: A Report from the Australian and New Zealand Fontan Registry. International Journal of Cardiology, 273, 100-107. https://doi.org/10.1016/j.ijcard.2018.07.118

[17] Elder, R.W., McCabe, N.M., Hebson, C., Veledar, E., Romero, R., Ford, R.M., Mahle, W.T., Kogon, B.E., Sahu, A., Jokhadar, M., McConnell, M.E. and Book, W.M. (2013) Features of Portal Hypertension Are Associated with Major Adverse Events in Fontan Patients: The VAST Study. International Journal of Cardiology, 168, 3764-3769. https://doi.org/10.1016/j.ijcard.2013.06.008

[18] Wallihan, D.B. and Podberesky, D.J. (2013) Hepatic Pathology after Fontan Palliation: Spectrum of Imaging Findings. Pediatric Radiology, 43, 330-338.

https://doi.org/10.1007/s00247-012-2531-y

[19] Nandwana, S.B., Olaiya, B., Cox, K., Sahu, A. and Mittal, P. (2018) Abdominal Imaging Surveillance in Adult Patients after Fontan Procedure: Risk of Chronic Liver Disease and Hepatocellular Carcinoma. Current Problems in Diagnostic Radiology, 47, 19-22. https://doi.org/10.1067/j.cpradiol.2017.04.002

[20] Saliba, T., Dorkhom, S., O’Reilly, E.M., Ludwig, E., Gansukh, B. and Abou-Alfa, G.K. (2010) Hepatocellular Carcinoma in Two Patients with Cardiac Cirrhosis. European Journal of Gastroenterology \& Hepatology, 22, 889-891. https://doi.org/10.1097/MEG.0b013e32832e2bec

[21] Asrani, S.K., Warnes, C.A. and Kamath, P.S. (2013) Hepatocellular Carcinoma after the Fontan Procedure. The New England Journal of Medicine, 368, 1756-1757. https://doi.org/10.1056/NEJMc1214222

[22] Elder, R.W., Parekh, S. and Book, W.M. (2013) More on Hepatocellular Carcinoma after the Fontan Procedure. The New England Journal of Medicine, 369, 490. https://doi.org/10.1056/NEJMc1306854

[23] Weyker, P.D., Allen-John Webb, C., Emond, J.C., Brentjens, T.E. and Johnston, T.A. (2014) Anesthetic Implications of Extended Right Hepatectomy in a Patient with Fontan Physiology. A \& A Case Reports, 2, 99-101. https://doi.org/10.1213/XAA.0000000000000012

[24] Rajoriya, N., Clift, P., Thorne, S., Hirschfield, G.M. and Ferguson, J.W. (2014) A Liver Mass Post-Fontan Operation. QJM, 107, 571-572.

https://doi.org/10.1093/qjmed/hcu020 
[25] Maeda, A., Shibata, S.C., Okitsu, K., Imada, T., Takahashi, A., Uchiyama, A., Kamibayashi, T. and Fujino, Y. (2015) Pain Management with Bilateral Continuous Thoracic Paravertebral Block in a Patient with Fontan-Associated Hepatocellular Carcinoma Undergoing Hepatectomy. Regional Anesthesia \& Pain Medicine, 40, 718-719. https://doi.org/10.1097/AAP.0000000000000316

[26] Kwon, S., Scovel, L., Yeh, M., Dorsey, D., Dembo, G., Krieger, E.V., Bakthavatsalam, R., Park, J.O., Riggle, K.M., Riehle, K.J. and Yeung, R.S. (2015) Surgical Management of Hepatocellular Carcinoma after Fontan Procedure. Journal of Gastrointestinal Oncology, 6, E55-E60.

[27] Yamada, K., Shinmoto, H., Kawamura, Y., Wakamatsu, H., Kawauchi, T., Soga, S., Ogata, S. and Kaji, T. (2015) Transarterial Embolization for Pediatric Hepatocellular Carcinoma with Cardiac Cirrhosis. Pediatrics International, 57, 766-770. https://doi.org/10.1111/ped.12619

[28] Takuma, Y., Fukada, Y., Iwadou, S., Miyatake, H., Uematsu, S., Okamoto, R., Sato, D., Matsukawa, H., Shiozaki, S., Kamada, M., Morito, T. and Araki, Y. (2016) Surgical Resection for Hepatocellular Carcinoma with Cardiac Cirrhosis after the Fontan Procedure. Internal Medicine, 55, 3265-3272. https://doi.org/10.2169/internalmedicine.55.6869

[29] Oh, C., Youn, J.K., Han, J.W., Kim, G.B., Kim, H.Y. and Jung, S.E. (2016) Hepatocellular Carcinoma after the Fontan Procedure in a 16-Year-Old Girl: A Case Report. Medicine (Baltimore), 95, e4823. https://doi.org/10.1097/MD.0000000000004823

[30] Josephus Jitta, D., Wagenaar, L.J., Mulder, B.J., Guichelaar, M., Bouman, D. and van Melle, J.P. (2016) Three Cases of Hepatocellular Carcinoma in Fontan Patients: Review of the Literature and Suggestions for Hepatic Screening. International Journal of Cardiology, 206, 21-26. https://doi.org/10.1016/j.ijcard.2015.12.033

[31] Dorsey, D., Kwon, S., Krieger, E., Yeung, R.S., Natrajan, K. and Dembo, G. (2016) Conflicting Hemodynamic Goals in an Adult Patient with Fontan Physiology Presenting for Resection of a Hepatocellular Carcinoma. Journal of Cardiothoracic and Vascular Anesthesia, 30, 452-454. https://doi.org/10.1053/j.jvca.2015.08.032

[32] Conroy, M.R. and Moe, T.G. (2017) Hepatocellular Carcinoma in the Adult Fontan Patient. Cardiology in the Young, 27, 407-409. https://doi.org/10.1017/S1047951116001360

[33] Martínez-Quintana, E., Monescillo, A. and Rodríguez-González, F. (2017) Hepatocellular Carcinoma in a Non-Failing Fontan Circulation. Revista Espanola de Enfermedades Digestives, 109, 375.

[34] Lo, K.S., Chan, M.Y., Ma, K.W., Tsang, S.H., Cheung, T.T. and Lo, C.M. (2018) Left Hepatectomy in a Patient with a Fontan Circulation. Translational Gastroenterology and Hepatology, 3, 51. https://doi.org/10.21037/tgh.2018.07.10

[35] Ashhab, A.A., Singh, D. and Debes, J.D. (2018) A Rare Cause of a Left Abdominal Liver Mass. Gastroenterology, 155, e12-e13. https://doi.org/10.1053/j.gastro.2017.11.287

[36] Mazzarelli, C., Cannon, M.D., Hudson, M., Heaton, N., Sarker, D., Kane, P., Quaglia, A. and Suddle, A. (2019) Hepatocellular Carcinoma as a Complication of Vascular Disease of the Liver after Fontan Procedure. Hepatology, 69, 911-913. https://doi.org/10.1002/hep.30194 